\title{
Dynamique de l'excrétion ovulaire de Schistosoma haematobium dans une cohorte de Kalifabougou (Mali) sous traitement par le praziquantel (PZQ) durant cinq ans
}

\author{
Dynamics of Egg Excretion of Schistosoma haematobium in a Longitudinal Cohort Under \\ Treatment with Praziquantel over a Five-Year Period in Kalifabougou, Mali
}

\author{
D. Safiatou Niaré $\cdot$ D. Doumtabe $\cdot$ A. Ongoiba $\cdot$ K. Sidibé $\cdot$ A. Traoré $\cdot$ J. Sangala $\cdot$ K. Kayentao $\cdot$ T.M. Tran $\cdot$ \\ P.D. Crompton · B. Traoré · O.K. Doumbo
}

Reçu le 4 septembre 2017 ; accepté le 27 février 2018

(C) Société de pathologie exotique et Lavoisier SAS 2018

Résumé L'objectif de cette étude était d'évaluer la dynamique de l'excrétion ovulaire de Schistosoma haematobium (Sh) après la mise à échelle du « traitement de masse » (TDM) avec le praziquantel (PZQ) de 2011 à 2016 dans une cohorte de volontaires vivant dans le village de Kalifabougou au Mali. Nous avons conduit une étude transversale sur 676 volontaires au mois de mai 2011 nichée dans une étude de cohorte de 695 volontaires, âgés de 3 mois à 25 ans et suivis de 2011 à 2016 . Les œufs de $S h$ ont été recherchés par la technique de filtration d'urines et ceux des géo helminthes et de Schistosoma mansoni avec le Kato-Katz. Le taux de couverture maximum de la population cible de Kalifabougou en TDM/PZQ était de $83 \%$ en 2015 et il n'a pas eu de TDM/PZQ en 2014. Le taux de prévalence de $S h$ montrait une réduction significative entre 2011, 2013 et 2014 avec respectivement 10,20\% [95\% CI $=10,04-$ 10,18]- 5,32\% [95\% CI $=5,30-5,33]$, et 5,25\% [95\% $\mathrm{CI}=5,24-5,31]$, suivi d'une augmentation à 10,60\% [95 \% CI $=10,47-10,63]$ en 2015 et d'une baisse significative en 2016 à 5,40 \% [95\% CI = 3,5-7,3]. Les enfants âgés de six à dix ans, et majoritairement les garçons, seraient plus infectés par $S h$, et pourraient servir de réservoir de parasites. Le TDM avec le PZQ reste une stratégie efficace pour le contrôle de la schistosomose à $S h$ à Kalifabougou. Des

D. Safiatou Niaré $\cdot$ D. Doumtabe $\cdot$ A. Ongoiba $\cdot$ K. Sidibé

A. Traoré · J. Sangala $\cdot$ K. Kayentao $\cdot$ B. Traoré

O.K. Doumbo $(\square)$

Malaria Research and Training Center/Département

d'Epidémiologie des Affections Parasitaires, LIG-MRTC-ICER,

FMPOS-FAPH, USTTB, Mali

e-mail : Sdoumbo@icermali.org; okd@icermali.org

T.M. Tran · P.D. Crompton

Laboratory of Immunogenetics/NIAID, 5601 Fishers Lane, MSC 9806, Bethesda, MD 20892-9806 (deliveries: Rockville, MD 20852), USA études complémentaires sur la couverture moyenne en TDM-PZQ, les comportements de contact homme-eau et les mouvements de population sont nécessaires pour comprendre la persistance du taux de prévalence annuel de $5 \%$ de l'excrétion ovulaire dans la cohorte de volontaires traités périodiquement par le PQZ. Un test de viabilité des œufs excrétés serait aussi une valeur ajoutée.

Mots clés Schistosoma haematobium · Prévalence · Cohorte $\cdot$ Traitement de masse $\cdot$ Praziquantel . Kalifabougou $\cdot$ Mali $\cdot$ Afrique intertropicale

Abstract This study aim was to evaluate the dynamics of Schistosoma haematobium eggs excretion after the scaling up of "Mass Drug Administration" (MDA) with praziquantel (PZQ) from 2011 to 2016 in a cohort of volunteers living in the village of Kalifabougou, Mali. We conducted a crosssectional study on 676 volunteers in May 2011 niched in cohort study from 696 volunteers aged three months to 25 years. The eggs of Schistosoma haematobium (Sh) were tested by urine filtration technique, Soil-transmitted helminth and Schistosoma mansoni by the Kato-Katz technique. Maximal MDA/ PZQ population coverage was $83 \%$ in 2015 and no MDA/PZQ n 2014. A total of 676 volunteers was included in this prospective cohort. The prevalence rate of Sh showed a significate decreasing from 2011, 2013 to 2014 with respectively $\quad 10.2 \% \quad[95 \% \quad \mathrm{CI}=10.04-10,18], \quad 5.32 \% \quad[95 \%$ $\mathrm{CI}=5.30-5.33]$, and $5.25 \%$ [95\% CI=524.-5.31], followed by an increase to $10.6 \%$ [ $95 \% \mathrm{CI}=10.47-10.63]$ in 2015 and a significative decrease in 2016 to $5.4 \%$ [95\% CI=3.5-7,3]. Children aged from six to 10 years and mostly boys were more infected with $\mathrm{Sh}$, then could serve of parasite reservoir. MDA with PZQ remains an effective strategy for schistosomiasis control against Sh in Kalifabougou. Additional studies on MDA/PZQ average treatment covering human-water 
contact behaviors and population migration are necessary to understand the persistence of the $5 \%$ annual prevalence rate of egg shedding in the cohort of volunteers periodically treated with PQZ. Testing eggs shed viability will be also an added value.

Keywords Schistosoma haematobium • Prevalence · Cohort · Mass Drug Administration · Praziquantel · Kalifabougou $\cdot$ Mali $\cdot$ Sub-Saharan Africa

\section{Introduction}

Au Mali, les schistosomoses sévissent de façon endémique dans toute la zone sahélienne et soudanienne nord pour Schistosoma haematobium (Sh) et de façon focalisée pour Schistosoma mansoni $(\mathrm{Sm})$. Dans la région de Koulikoro et dans le district sanitaire de Kati, les zones d'endémie sont Kambila et Kati [7,27]. Dans le village de Kalifaboubou, le taux de prévalence initial de la schistosomose à $S h$ était de $12,7 \%$ en 2005 (données non publiées du Programme national de lutte contre les schistosomoses PNLSH au Mali).

Le pays dispose depuis 1982 d'un PNLSH avec les stratégies suivantes : la chimiothérapie à base de praziquantel (PZQ), l'amélioration de l'accès aux soins, l'accès à l'eau potable et aux latrines et l'éducation pour la santé $[1,6]$.

La stratégie intégrée de la campagne du traitement de masse (TDM) à base communautaire par le PZQ couplée à l'albendazole (ABZ) et l'ivermectine (IVER) a démarré en 2005 suite à la politique d'élimination des schistosomoses et des géohelmintes au niveau national [8]. La mise à échelle du TDM (PZQ, ABZ, IVER) dans le village de Kalifabougou a démarré en 2010. Les taux de couverture des populations cibles par ces stratégies étaient de 72,0\%, 52,3\%, $59,6 \%, 0 \%, 83 \%, 72 \%$ respectivement en 20112012 , 2013, 2014, 2015, 2016.

Le « Malaria Research and Training Center» du Département d'épidémiologie des affections parasitaires et ses partenaires du National Institutes of Health (NIH) USA ont mis en place au sein du Centre de santé communautaire (CSCOM) de Kalifabougou un centre de formation et de recherche sur le paludisme et les co-infections avec les helminthes en 2011. Nous suivons depuis une cohorte de 695 volontaires âgés de 3 mois à 25 ans. L'objectif initial de cette étude de cohorte était de comprendre les mécanismes d'acquisition de l'immunité naturelle antimalarique en zone d'endémie palustre. L'objectif secondaire était d'analyser l'impact de la co-infection schistosome-plasmodium dans cette acquisition. Ce qui a amené à réaliser systématiquement des examens de selles et d'urines, à chaque passage transversal annuel. Nous avons pu évaluer de 2011 à 2016 l'incidence annuelle et l'évolution des prévalences des infestations et la charge d'excrétion ovulaire des deux schistosomoses $(S h, S m)$ dans cette cohorte.

\section{Sujets et méthodes}

\section{Site d'étude}

Le village de Kalifabougou est situé à $40 \mathrm{~km}$ au nord de Bamako, la capitale, en zone de savane soudanienne nord avec les coordonnées suivantes $12^{\circ} 56^{\prime} 39^{\prime \prime} \mathrm{N}$ et $8^{\circ} 10^{\prime} 15^{\prime}$ W. Durant les cinq années d'étude, il est tombé de 800 à $1200 \mathrm{~mm}$ de pluie par an dans le village de Kalifabougou (données du service de la météorologie nationale de 2011 à 2016).

Le village est traversé par une rivière temporaire, située à proximité de l'école et des résidences, qui assure les besoins en eau (toilette, lavage du linge et des ustensiles de cuisine, baignade) et de maraichage de la population, en saison de pluies et en saison sèche froide, de juin à décembre. En dehors de cette source d'eau de surface, la population dispose de puits traditionnels pour l'approvisionnement en eau potable (boisson, cuisine) et un forage réalisé au mois de mai 2017. Il n'y a pas d'autres sources d'eau de surface (marigots, céanes, retenues) pouvant héberger des hôtes intermédiaires de schistosomes.

La population est majoritairement agricole avec quelques activités pastorales et de commerce. Le village dispose d'une école de neuf classes et d'un lycée construit en 2013. La population d'élèves s'élevait en 2013 à 1525 élèves (738 au premier cycle, 485 au second cycle et 302 au lycée). Ces élèves ont des activités de contact homme-eau (baignade) pendant les périodes de recréation, de pauses déjeuner, de week-end et de vacances.

Les taux de prévalence de base de l'excrétion ovulaire de schistosomes dans la population de Kalifabougou étaient de $12,7 \%(483 / 3800)$ en 2005 et dans les autres communes de Kati de 12,9 \% (903/7000) (données du PNLSH du Mali). Nous ne disposons pas d'étude sur les hôtes intermédiaires de schistosomes.

Dans le cadre de la politique nationale d'élimination des schistosomes et de géohelminthes, une mise à échelle des TDM a été lancée dans tout le pays, quel que soit le niveau d'endémicité. Le programme de TDM au PZQ a démarré effectivement dans le village de Kalifabougou en 2010 avec un taux de couverture initial de la population cible de $52 \%$ (données CSCOM 2010). La majorité des enfants d'âge scolaire a reçu du PZQ, de l'albendazole et de l'ivermectine au mois de mars 2011, ainsi que les volontaires de notre cohorte avant leur enrôlement dans l'étude [17,23].

Les passages transversaux pour la recherche de schistosomes et de géohelminthes dans notre cohorte étaient nichés 
dans l'étude mère sur la dynamique de l'acquisition de l'immunité naturelle antimalarique dans le village de Kalifabougou. Nous avons utilisé cette opportunité pour évaluer la dynamique de l'excrétion ovulaire des schistosomes dans cette cohorte, suite à la prise annuelle de PZQ. De 2011 à 2016, nous avons réalisé systématiquement des examens de selles et d'urines chez chaque volontaire.

La population cible était constituée des enfants et jeunes adultes de âgés de trois mois à vingt-cinq ans vivant depuis plus d'un an à Kalifabougou. La taille de l'échantillon de 676 volontaires a été calculée pour l'étude d'acquisition naturelle de l'immunité antimalarique, avec un risque alpha de $5 \%$ et une puissance 1-bêta de $80 \%$ [24]. Les volontaires ont été sélectionnés par échantillonnage aléatoire dans chaque strate d'âge sur la base d'un recensement de l'ensemble de la population du village. Les détails de la sélection aléatoire des volontaires de la cohorte sont décrits dans les publications précédentes $[24,25]$. Six cent soixante-seize (n $=676$ ) volontaires ont rempli les différents critères d'inclusion et d'exclusion pour l'étude mère sur l'acquisition de l'immunité anti-palustre [24,25]

Après l'étape de la permission communautaire, nous avons convoqué les volontaires par famille pour l'obtention d'un consentement éclairé écrit pour les prélèvements de selles et d'urines pour la recherche d'œufs de schistosomes et de géohelmintes.

\section{Collecte et traitement des échantillons}

Chaque volontaire consentant a reçu deux pots de prélèvement pour la collecte des selles et des urines, portant son numéro d'étude. Les échantillons d'urines ont été collectés entre $9 \mathrm{~h}$ et $14 \mathrm{~h}$. Pour la recherche des œufs de $S h$, un volume de $10 \mathrm{ml}$ a été analysé pour chaque volontaire, par la méthode de filtration. Pour la visualisation des œufs de $S h$, les filtres ont été colorés par la nynhidrine à $5 \%$ et comptés au microscope optique à l'objectif x 10 puis x 40 [5]

Les selles ont été examinées par la technique semiquantitative de Kato-Katz [3], pour les œufs de Sm et de géohelminthes.

\section{Considérations éthiques}

Le protocole de recherche a été approuvé par le comité d'éthique institutionnel de la Faculté de médecine, de pharmacie et d'odonto-stomatologie (FMPOS) du Mali et celui du NIH. (ClinicalTrials.gov identifier: NCT01322581). Le consentement libre et éclairé des volontaires adultes et des parents /gardiens des enfants a été obtenu (devant un témoin), signé et documenté.

Tous les volontaires excréteurs d'œufs de schistosomes ont été traités systématiquement avec le PZQ selon la politique du Programme national de lutte contre les schistosomoses au Mali (qu'ils aient reçu ou pas le TDM-PZQ populationnel).

\section{Collecte, gestion et analyse statistique des données}

Les données cliniques et biologiques des volontaires ont été reportées dans un questionnaire et saisies dans un fichier Excel. L'analyse des données a été faite par le logiciel SPSS version 16.0. Le test $t$ de Student et de l'ANOVA ont été utilisés pour comparer les moyennes des œufs. Le test Chi2 de Pearson (avec la correction de Yates, si besoin) et/ou de MacNemar a été utilisé pour comparer les taux de prévalence. Le seuil de signification a été fixé à 0,05

\section{Résultats}

Un total de 676 volontaires a été inclus dans la cohorte au mois de mai 2011 et suivi jusqu'en 2016. Le taux de suivi de la cohorte était de $90 \%$ jusqu'en 2016.

\section{Caractéristiques sociodémographiques des volontaires}

La moyenne d'âge à l'inclusion était de 7,7 $( \pm 4,2)$ ans en mai 2011 et le sex-ratio de 1,03 en faveur du sexe masculin. Le tableau 1 montre la distribution de la moyenne des âges par année de 2011 à 2016, avec une différence statistiquement significative par le test de ANOVA $(p<0,001)$.

Tableau 1 Distribution de la moyenne des âges des volontaires de 2011 à 2016 dans le village de Kalifabougou / Volunteers mean age distribution from 2011 to 2016 in Kalifabougou village

\begin{tabular}{|lllllll|}
\hline Années & $\mathbf{2 0 0 1}$ & $\mathbf{2 0 1 3}$ & $\mathbf{2 0 1 4}$ & $\mathbf{2 0 1 5}$ & $\mathbf{2 0 1 6}$ \\
& $\mathbf{n}=\mathbf{6 7 6}$ & $\mathbf{n = 5 6 4}$ & $\mathbf{n = 5 3 3}$ & $\mathbf{n}=\mathbf{5 0 7}$ & $\mathbf{n = 5 2 0}$ \\
\hline Âge moyen & 7,77 & 7,83 & 7,49 & 8,11 & 7,73 \\
Écart type & 4,2 & 4,13 & 3,78 & 5,02 & 7,07 & 1,13 \\
Sex ratio & 1,03 & 1,13 & 1,15 & 1,2 & \\
\hline
\end{tabular}


La couverture en PZQ au cours des traitements de masse à base communautaire à Kalifabougou de 2012 à 2016 est présentée dans le tableau 2

\section{Evolution du taux d'excrétion ovulaire de 2011 à 2016}

Le taux de prévalence de $S$. haematobium était de 10,2\% $(69 / 676)[95 \% \mathrm{CI}=10,04-10,18]$ à l'inclusion en 2011. Cette prévalence a subi une réduction significative de 2011 à 2014 : elle était de 5,32\% [ $95 \% \mathrm{CI}=5,30-5,33]$, et 5,25\% [95\% CI $=5,19-5,31]$ respectivement en 2013 et $2014(\mathrm{p}<$ $0,001)$. Elle a doublé en 2015 à 10,5\%, [95\% CI $=10,47$ $10,63]$, puis a subi une baisse significative en 2016 revenant à $5,4 \%[95 \% \mathrm{CI}=3,5-7,3]$.

\section{Comparaison des taux de prévalence par tranches d'âges}

La figure 1 montre que les enfants de 6-10 ans étaient plus infectés (quelle que soit l'année), comparés aux autres grou- pes, avec une différence statistiquement significative $(p=0,009)$. Les taux de prévalence des autres tranches d'âges étaient comparables d'une année à l'autre, mais faibles $(p>0,05)$ (Fig. 1).

\section{Comparaison des taux de prévalence par sexe}

Les résultats de la figure 2 montrent que les volontaires du sexe masculin étaient plus infectés que ceux du sexe féminin en 2011 et $2015(p=0,007)$. Mais cette différence n'était pas statistiquement significative les autres années, 2012, 2013, 2014 et $2016(\mathrm{p}=0,82)$.

\section{Comparaison des charges ovulaires de $S h$ par tranches d'âges}

La charge ovulaire était plus élevée chez les enfants de la tranche d'âge de 6-10 ans, comparés aux autres groupes (Tableau 3).

\begin{tabular}{|c|c|c|c|c|}
\hline Année & Population totale & Nombre ciblé & Nombres atteint & $\begin{array}{l}\text { Taux de couverture } \\
\text { (\%) }\end{array}$ \\
\hline Mars 2001 & 14001 & 5600 & 4032 & 72 \\
\hline Juin 2012 & 14210 & 5620 & 2940 & 52,3 \\
\hline Juin 2013 & 14980 & 5600 & 3340 & 59,6 \\
\hline Juin 2015 & 15741 & 6200 & 5140 & 83 \\
\hline Nov. 2016 & 16944 & 6780 & 4889 & 72 \\
\hline
\end{tabular}

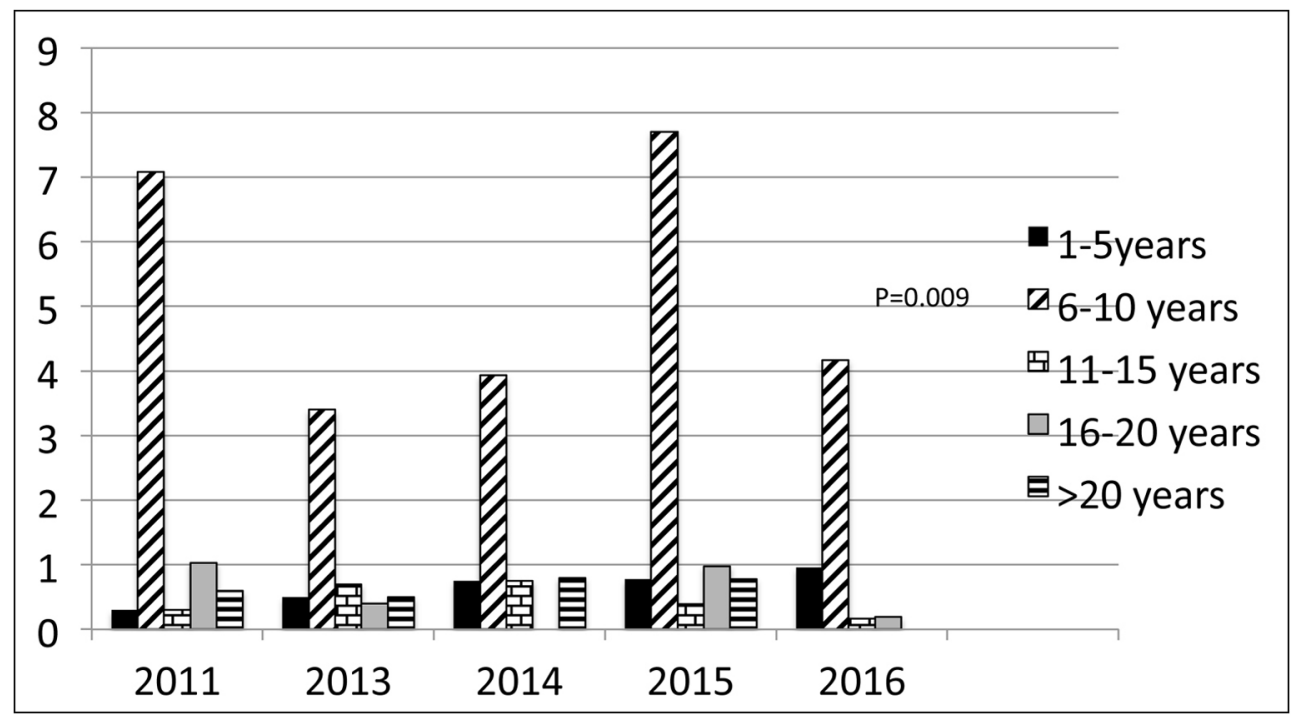

Fig.1 Évolution des taux de prévalences de l'excrétion ovulaire de S.h. suivant l'âge dans le village de Kalifabougou de 2011 à 2016 / Evolution of the prevalence rate of S.h. eggs excretions according to the age in Kalifabougou village 


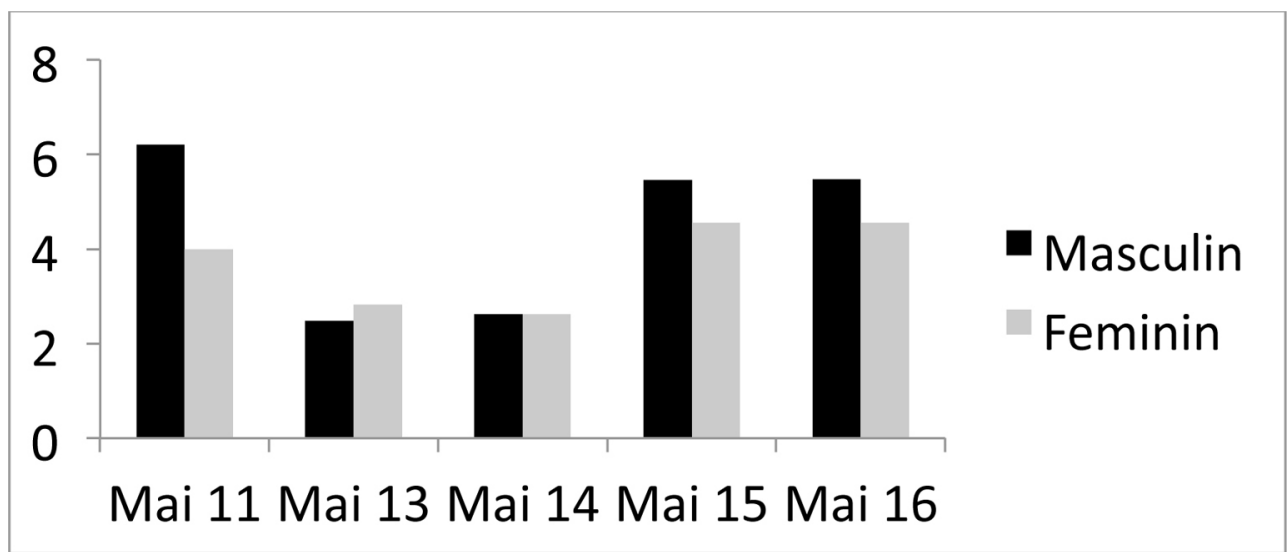

Fig.2 Comparaison des taux d'excrétion ovulaire de S. haematobium selon le sexe dans le village de Kalifabougou / Comparison of Schistosoma haematobium eggs excretion rate according to gender in Kalifabougou village

\begin{tabular}{|c|c|c|c|c|c|c|}
\hline Année & Cufs & $\begin{array}{l}0-5 \text { ans } \\
\text { n (\%) }\end{array}$ & $\begin{array}{l}\text { 6-10 ans } \\
\text { n (\%) }\end{array}$ & $\begin{array}{l}11-15 \text { ans } \\
\text { n (\%) }\end{array}$ & $\begin{array}{l}16-20 \text { ans } \\
\text { n (\%) }\end{array}$ & $\begin{array}{l}21-25 \text { ans } \\
\text { n (\%) }\end{array}$ \\
\hline \multirow[t]{2}{*}{2011} & $<50$ œufs & $2(0,3)$ & $50(7,4)$ & $2(0,3)$ & $7(1)$ & $3(0,4)$ \\
\hline & $\geq 50$ œufs & 0 & $4(0,6)$ & 0 & 0 & $1(0,1)$ \\
\hline \multirow[t]{2}{*}{2013} & $<50$ œufs & $2(0,3)$ & $17(3)$ & $4(0,7)$ & $1(0,2)$ & $3(0,5)$ \\
\hline & $\geq 50$ œufs & 0 & $2(0,3)$ & 0 & $1(0,2)$ & 0 \\
\hline \multirow[t]{2}{*}{2014} & $<50$ œufs & $3(0,6)$ & $18(3,4)$ & $4(0,8)$ & 0 & $1(0,2)$ \\
\hline & $\geq 50$ œufs & 0 & $3(0,6)$ & 0 & 0 & 0 \\
\hline \multirow[t]{2}{*}{2015} & $<50$ œufs & $4(0,8)$ & $34(6,7)$ & $2(0,4)$ & $3(0,6)$ & $4(0,8)$ \\
\hline & $\geq 50$ œufs & 0 & $5(1)$ & 0 & $2(0,4)$ & 0 \\
\hline \multirow[t]{2}{*}{2016} & $<50$ œufs & $5(0,9)$ & $16(3,1)$ & $1(0,2)$ & 0 & 0 \\
\hline & $\geq 50$ œufs & 0 & 0 & 0 & $1(0,2)$ & 0 \\
\hline
\end{tabular}

Nous n'avons pas trouvé de portage chronique de schistosomes chez nos volontaires au cours du suivi. Onze cas de réinfection ont été observés : trois cas en 2013, deux cas en 2014, trois cas en 2015 et six cas en 2016. Nous n'avons pas non plus trouvé d'excréteur d'œufs de $S h$ chez les nourrissons de moins d'un an dans ce village.

Un cas isolé de portage de $S$. mansoni a été détecté en 2011. Nous n'avons pas trouvé d'œufs de géohelmintes.

\section{Discussion}

Nous avons utilisé l'opportunité d'un suivi de cohorte pour l'acquisition de l'immunité naturelle antimalarique, pour évaluer l'impact d'un TDM-PZQ populationnel et d'un « screen and treat » dans une cohorte de population cible de âgée de trois mois à vingt-cinq ans, dans un village d'endémie modérée de $S h$. Le calcul de la taille de l'échantillon n'a pas été fait sur la base du taux de prévalence de base de Sh. Le taux de suivi de la cohorte sur six ans est correct
(90\%). Les perdus de vus étaient justifiés (grossesses, voyages). Nous pensons que cette évaluation peut être valide statistiquement et représentative pour les résultats trouvés, afin d'informer le PNLSH au Mali.

Au cours de cette étude, sur un total de 676 volontaires inclus dans une cohorte en 2011, la prévalence de la schistosomose à $S h$ a subi une réduction significative de 2011 à 2014 et une augmentation en 2015, suivie d'une diminution en 2016. Cette augmentation du nombre d'excréteurs d'œufs de $S h$ en 2015 s'explique par la rupture d'approvisionnement du PZQ au niveau national. L'absence de TDM-PZQ à Kalifabougou en 2014 a influencé le taux de réinfection en 2015. Ce qui montre que le traitement systématique des sujets positifs lors de l'enquête transversale de 2014 n'a pas eu d'effet sur la transmission de Sh dans notre cohorte. La reprise du TDM-PZQ en 2015 a entraîné une réduction significative en $2016(5 \%)$.

Toutefois la schistosomose urinaire à $S h$ reste présente à Kalifabougou, avec un niveau faible d'excrétion ovulaire résiduel annuel de $5 \%$ en 2012, 2013, 2014 et 2016. Il faut 
noter qu'il n'y a pas eu de portage chronique de $S h$ de 2011 à 2016 à Kalifabougou, sous TDM-PZQ et « screen and treat » annuel. Ce qui est en faveur de l'existence d'excréteurs d'œufs de $S h$ à Kalifaboubou ayant échappé au TDM. Le taux moyen de couverture en TDM-PZQ $(<80 \%$ de la population cible) expliquerait cette transmission résiduelle. Une étude malacologique complète (espèces de mollusques présents, taux d'infestation en cercaires), de viabilité des œufs des excréteurs résiduels et de comportement de contact Homme-eau permettrait d'expliquer cette persistance de la transmission.

La tranche d'âge 6-10 ans est la plus infectée $(p=0,009)$ avec une charge parasitaire plus élevée. Le sexe masculin est aussi plus infesté que le sexe féminin. Cette tranche d'âge pourrait constituer le réservoir de $S h$ dans Kalifabougou. Un traitement de masse ciblé sur cette tranche d'âge, couplé à une stratégie de « screen and treat », pourrait être une approche efficiente dans ces zones d'endémicité modérée.

Les taux de prévalence élevés chez les garçons sont retrouvés dans plusieurs études $[4,20,22]$. Ceci s'explique en partie par les facteurs socioculturels et comportementaux (abreuvage des animaux par les bergers, jeux, baignades, maraichages) qui exposent plus les garçons au contact avec l'eau que les filles $[4,10,12,13]$.

Nous avons utilisé une approche pragmatique, opportuniste, en intégrant une étude de cohorte en cours dans ce village. Nous n'avons pas recherché systématiquement les signes physiques, radio-échographiques et fonctionnels de $S h$ chez les volontaires.

L'absence d'examens échographiques ne nous permet pas de nous prononcer sur la présence ou non de complications rénales et vésicales.

La diminution des taux de prévalence de $S h$ à Kalifabougou de 2011 à 2014 reste comparable à celle obtenue par Togola et al., entre 2011 et 2014 lesquels montraient une diminution des taux de prévalence de la schistosomose à Sh à Bamako (sur la rive gauche du Niger) passant de $17,4 \%$ à 13,8 \% ainsi qu'à Sotuba après le TDM à base de praziquantel $[21,23]$. Toutefois, Sangho et al ainsi que Dabo et al n'ont pas trouvé cette différence entre les garçons et les filles en milieu urbain de Bamako [7,14,19].

Les garçons de 6-10 ans pourraient constituer un réservoir de parasites dans le village, nécessitant un traitement ciblé (Mass Screening And Treat - MSAT) pour aller vers l'élimination des $S h$ dans la population de Kalifabougou [10].

Au Sénégal, la même tendance a été observée par Senghor, et al après un traitement par une dose de PZQ de $40 \mathrm{mg} / \mathrm{kg}$ par poids corporel avec une réduction de l'excrétion ovulaire de 73,2 \% à 4,6\% [20]. Des études antérieures avaient aussi montré une efficacité élevée du PZQ avec une réduction significative du taux d'excrétion ovulaire dans les urines dans les zones d'intense transmission de $S h$ [15,17]. La réduction du taux de prévalence a été aussi décrite par plusieurs auteurs qui montraient aussi que malgré l'efficacité de PZQ sur $S h$, la réinfection peut survenir rapidement au sein d'une même population endémique et atteindre le niveau du prétraitement $[11,13,16]$.

L'absence de portage chronique chez les volontaires ne nous permet pas actuellement de poser l'hypothèse de la résistance des souches de $S h$ au PZQ, dans la zone de Kalifabougou bien qu'une baisse de l'efficacité du PZQ ait été observée au cours d'études antérieures [9]. Ce qui renforce l'intérêt d'une étude rigoureuse sur la sensibilité des schistosomes au PZQ dans la population de Kalifabougou.

Les études ont montré que les schistosomules ne sont pas sensibles aux PZQ [20] : elles pourraient donc évoluer au stade adulte après le traitement TDM-PZQ : ainsi les porteurs pourraient constituer des réservoirs de parasites. La mort du ver adulte ne pouvant pas être mesurée directement en raison de sa localisation intravasculaire, l'efficacité du PZQ est actuellement estimée à l'aide de la clairance urinaire des œufs, trois à quatre semaines après le traitement [15].

Des biomarqueurs ayant une valeur pronostique potentielle peuvent être aussi mesurés par des essais d'antigène de schistosome mis au point à l'aide de sérums ou d'urine [26].

La schistosomose à $S$. mansoni n'est pas endémique dans le village de Kalifabougou. Nos résultats montrent un seul cas de $S m$ sur 676 volontaires dépistés en 2011. Depuis, aucun autre cas n'a été détecté les années suivantes chez les participants. Dans le cercle de Kati, aucune étude n'a montré la présence de $S m$. Le seul cas observé chez un garçon de huit ans pourrait être considéré comme un cas importé dans une population sujette à des déplacements fréquents en dehors de Kalifabougou. Le participant a été traité par le PZQ et nous n'avons plus détecté de $S m$ durant le reste de son suivi. L'absence de $S m$ dans le village de Kalifabougou pourrait être expliquée par le caractère non permanent de la rivière qui traverse le village. Les hôtes intermédiaires de $S m$ ne résistent pas à la sècheresse (anhydrobiose). Contrairement aux bulins, hôte intermédiaire de Sh, Biomphalaria pfeifferi colonise les collections d'eaux claires sur fond de sable et gravier, eaux stagnantes ou animées d'un très léger courant, avec une végétation aquatique abondante [18,28].

Malgré ses limites, les résultats de cette recherche peuvent éclairer le PNLSH sur l'impact de leurs stratégies de contrôle, voire d'élimination de la schistosomose au Mali.

\section{Conclusion}

La schistosomose à S. haematobium reste modérément endémique dans le village de Kalifabougou. Les garçons de 610 ans constitueraient les potentiels réservoirs de parasites. La couverture en TDM-PZQ reste moyenne et laisser échapper des excréteurs d'œufs de $S h$. 
La stratégie de dépistage ciblé et les traitements au PZQ pourraient être plus efficients dans les zones d'endémicité modérée, comme Kalifabougou.

Remerciements Nous remercions le NIAID du NIH qui a financé cette étude. Nos remerciements s'adressent également au responsable du PNLCS, au médecin chef du centre de santé communautaire de Kalifabougou et à tout le personnel ainsi qu'au chef de village et à la population d'étude de Kalifabougou.

Liens d'intérêt : Les auteurs déclarent ne pas avoir de liens d'intérêt.

\section{References}

1. Ahmed AM, Abbas H, Mansour FA, et al (2012) Schistosoma haematobium infections among schoolchildren in central Sudan one year after treatment with praziquantel. Parasites Vectors 5:108. doi: 10.1186/1756-3305-5-108.

2. Botros S, Pica-Mattoccia L, William S, et al (2005) Effect of praziquantel on the immature stages of Schistosoma haematobium. Int J Parasitol 35:1453-7

3. Bustinduy AL, Sousa-Figueiredo JC, Adriko M, et al (2013) Fecal occult blood and fecal calprotectin as point-of-care markers of intestinal morbidity in Ugandan children with Schistosoma mansoni infection. PLoS Negl Trop Dis 7:e2542. doi: 10.1371/journal.pntd.0002542

4. Campbell SJ, Stothard JR, O'Halloran F, et al (2017) Urogenital schistosomiasis and soil-transmitted helminthiasis (STH) in Cameroon: An epidemiological update at Barombi Mbo and Barombi Kotto crater lakes assessing prospects for intensified control interventions. Infect Dis Poverty 6:49. doi: 10.1186/s40249-017-0264-8

5. Cheesbrough M (1987) Medical laboratory manual for tropical countries. Elsevier Health Sciences, $624 \mathrm{p}$

6. Colley DG, Bustinduy AL, Secor E, King CH (2014) Human schistosomiasis. Lancet 383:2253-64. doi: 10.1016/S0140-6736(13)61949-2

7. Dabo A, Badawi HM, Bary B, Doumbo OK (2011) Urinary schistosomiasis among preschool-aged children in Sahelian rural communities in Mali. Parasit Vectors 4:21. doi: 10.1186/1756-3305-4-21.

8. Dembele M, Bamani S, Dembele R, et al (2012) Implementing preventive chemotherapy through an integrated National Neglected Tropical Disease Control Program in Mali. PLoSNegl Trop Dis 6: e1574

9. Doenhoff MJ, Cioli D, Utzinger J (2008) Praziquantel: mechanisms of action, resistance and new derivatives for schistosomiasis. Curr Opin Infect Dis 21:659-67. doi: 10.1097/QCO.0b013e328318978f

10. Global Burden of Disease Study 2013 Collaborators (2015) Global, regional, and national incidence, prevalence, and years lived with disability for 301 acute and chronic diseases and injuries in 188 countries, 1990-2013: a systematic analysis for the Global Burden of Disease Study 2013. Lancet 386:743-800. doi: 10.1016/S0140-6736(15)60692-4

11. Global Link for Online Biomedical Expertise (2017) [http://www. globe-network.org]

12. Hotez PJ, Kamath A (2009). Neglected tropical diseases in subsaharan Africa: review of their prevalence, distribution, and disease burden. PLoSNegl Trop Dis 3:e412. doi: 10.1371/journal. pntd.0000412

13. Kabatereine NB, Brooker S, Koukounari A, et al (2007) Impact of a national helminth control programme on infection and morbidity in Ugandan schoolchildren. Bull World Health Organ 85:91-9

14. Landouré A, Dembélé R, Goita S, et al (2012) Significantly reduced intensity of infection but persistent prevalence of schistosomiasis in a highly endemic region in Mali after repeated treatment. PLoS Negl Trop Dis 6: e1774. doi: 10.1371/journal. pntd.0001774

15. Mutapi F, Rujeni N, Bourke C, et al (2011) Schistosoma haematobium treatment in 1-5 year old children: safety and efficacy of the antihelminthic drug praziquantel. PLoS Negl Trop Dis 5: e1143. doi: 10.1371/journal.pntd.0001143

16. Norton AJ, Gower CM, Lamberton PH, et al (2010) Genetic consequences of mass human chemotherapy for Schistosoma mansoni: population structure pre- and post-praziquantel treatment in Tanzania. Am J Trop Med Hyg 83:951-7. doi: 10.4269/ajtmh.2010.10-0283

17. OMS (2017) Schistosomiase (bilharziose) Aide-mémoire [http:// www.who.int/mediacentre/factsheets/fs115/fr/]

18. Rollinson D (2010) Biomphalaria: Natural History, Ecology and Schistosome Transmission in: Biomphalaria Snails and Larval Trematodes, Springer, pp 57-797

19. Sangho H, Dabo A, Sangho O, et al (2005) Prévalence et perception de la schistosomose en zone de riziculture irriguée au mali. Mali Méd T 20:15-20

20. Senghor B, Diallo A, Sylla SN, et al (2014) Prevalence and intensity of urinary schistosomiasis among school children in the district of Niakhar, region of Fatick, Senegal. Parasites Vectors. 7:5. doi: 10.1186/1756-3305-7-5.

21. Tandina F, Doumbo SN, Kone AK, et al (2016). Épidémiologie des schistosomoses dans le village périurbain de Sotuba, dix années après la mise à échelle du traitement de masse au Mali. Médecine et Santé Tropicales 26:51-6

22. Taqua OH, Haitham MA El-Bingawi (2012) Epidemiological survey: Schistosoma haematobium in schoolchildren of White Nile areas, Khartoum. Sudan Med J 48:135-40

23. Togola S (2015) Evolution de la prévalence et de l'intensité de 2011 à 2014 en milieux scolaires du district de Bamako, Mali, FMPOS, Bamako, Thèse de Médecine

24. Tran TM, Li S, Doumbo S, et al (2013) An intensive longitudinal cohort study of malian children and adults reveals no evidence of acquired immunity to Plasmodium falciparum infection. Clin Infect Dis 57:40-7. doi: 10.1093/cid/cit174

25. Tran TM, Ongoiba A, Coursen J, et al (2014) Naturally acquired antibodies specific for Plasmodium falciparum reticulocytebinding protein homologue 5 inhibit parasite growth and predict protection from malaria. J Infect Dis 209: 789-798

26. Van Dam GJ, de Dood CJ, Lewis M, et al (2013). A robust dry reagent lateral flow assay for diagnosis of active schistosomiasis by detection of Schistosoma circulating anodic antigen. Exp Parasitol 135:274-82. doi: 10.1016/j.exppara.2013.06.017

27. Webster BL, Diaw OT, Seye MM, et al (2013) Praziquantel treatment of school children from single and mixed infection foci of intestinal and urogenital schistosomiasis along the Senegal River Basin: monitoring treatment success and re-infection patterns. Acta Trop128:292-302. doi: 10.1016/j.actatropica.2012.09.010

28. Woolhouse MEJ (1992) Population Biology of the Freshwater Snail Biomphalaria pfeifferi in the Zimbabwe Highveld. Journal of Applied Ecology 29:687-94. doi: 10.2307/2404477 